\title{
Riemann problem for a compressible perfect fluid with a constant external force for the Chaplygin gas
}

Yicheng Pang ${ }^{1 *}, \mathrm{Min} \mathrm{Hu}^{1}$ and Jinhuan Wang ${ }^{2}$

${ }^{*}$ Correspondence:
ypanggy@outlook.com
1School of Mathematics and
Statistics, Guizhou University of
Finance and Economics, Guiyang,
China
Full list of author information is
available at the end of the article

*Correspondence:

ischool of Mathematics and

Statistics, Guizhou University of

China

available at the end of the article

\begin{abstract}
The Riemann problem for a compressible perfect fluid with a constant external force for the Chaplygin gas is considered. We obtain two kinds of exact solutions. The first one consists of contact discontinuities, while the other one involves a delta shock wave in which both density and internal energy contain a Dirac delta function. The position, speed and weights of the delta shock wave are derived from both generalized Rankine-Hugoniot relation and entropy condition, which are established in detail. Moreover, the solutions are no longer self-similar due to the influence of the constant external force.
\end{abstract}

Keywords: Delta shock wave; Compressible fluids; Riemann problem; Source term

\section{Introduction}

The one-dimensional compressible perfect fluid is governed by

$$
\left\{\begin{array}{l}
\rho_{t}+(\rho u)_{x}=0, \\
(\rho u)_{t}+\left(\rho u^{2}+p\right)_{x}=f \rho, \\
\left(\rho u^{2} / 2+\rho e\right)_{t}+\left(\left(\rho u^{2} / 2+\rho e+p\right) u\right)_{x}=f \rho u
\end{array}\right.
$$

where the $\rho, u, p, e$ denote density, velocity, pressure and specific energy, and $f$ represents a specific external force, such as gravity, Coriolis and electromagnetic forces [1,2]. The pressure $p$ and specific energy $e$ are given functions of the density $\rho$ and the specific entropy $s$, satisfying the thermodynamical constraint

$$
T \mathrm{~d} s=\mathrm{d} e+p \mathrm{~d} \frac{1}{\rho}
$$

with $T=T(\rho, s)$ being the temperature. In this paper, we are interested in a perfect fluid characterized by the equation of state

$$
p=-\frac{1}{\rho}
$$

which is called the Chaplygin gas. It was introduced by Chaplygin [3], Tsien [4] and von Karman [5] as a suitable approximation for calculating the lifting force on a wing of an

(c) The Author(s) 2018. This article is distributed under the terms of the Creative Commons Attribution 4.0 International License (http://creativecommons.org/licenses/by/4.0/), which permits unrestricted use, distribution, and reproduction in any medium, provided you give appropriate credit to the original author(s) and the source, provide a link to the Creative Commons license, and indicate if changes were made. 
airplane in aerodynamics. The Chaplygin gas possesses a negative pressure and describes a transition from a decelerated cosmological expansion to a stage of cosmic acceleration. Such a gas was also advertised as a possible model of dark energy [6-8].

The first and second equations of system (1.1) consist of the model

$$
\left\{\begin{array}{l}
\rho_{t}+(\rho u)_{x}=0 \\
(\rho u)_{t}+\left(\rho u^{2}+p\right)_{x}=f \rho .
\end{array}\right.
$$

If $f=0$, the solutions with concentration to the Riemann problem for (1.4) with the Chaplygin gas were obtained by Brenier [9]. Guo, Sheng and Zhang [10] solved completely this problem, where the delta shock wave solutions were constructed. Roughly speaking, the delta shock wave solution is a solution such that at least one of the variables contains Dirac delta function [11-16]. Besides, Wang [17] obtained the solutions to the Riemann problem for system (1.4) with the generalized Chaplygin gas, and the formation of delta shock wave was analyzed. The limit behavior of these solutions was clarified as the pressure vanishes by Sheng, Wang and Yin [18]. Yang and Wang [19] studied further system (1.4) for the modified Chaplygin gas, and the limit behavior of constructed Riemann solutions was analyzed as the pressure vanishes. The reader is also referred to [12, 20-22] and the references therein for related studies of system (1.4) with $f=0$. For the case that $f=\beta$ is a constant, Shen [23] and Sun [24] constructed, respectively, the Riemann solutions to system (1.4) for the Chaplygin gas and the generalized Chaplygin gas, while the influence of the external force on these solutions was displayed. In addition, a global weak solution to the Cauchy problem for system (1.4) was obtained by using the vanishing artificial viscosity and the compensated compactness theory [25]. However, only little work has contributed to the investigations of system (1.1).

For analyzing the energy transport processes of a fluid, it is necessary to consider system (1.1). Kraiko [26] studied the Riemann problem for system (1.1) with $f=0$ and $p=0$, and the discontinuities which carry mass, impulse and energy, were used to construct the solutions for arbitrary initial data. To avoid the definition of products of distributions, Nilsson, Rozanova and Shelkovich [27, 28] also considered this problem by introducing the internal energy $H=\rho e$, and they have shown the processes of concentration of both mass and internal energy on the delta shock wave front. Subsequently, this problem was solved completely by Cheng [29], and the delta shock wave with Dirac delta function in both the density and the internal energy was found in the solutions. The reader may refer to [30-34] and the references cited therein for further studies on the delta shock wave with Dirac delta function in multiple state variables. If $f=0$, the solutions to the Riemann problem for system (1.1) with the Chaplyin gas were constructed by Zhu and Sheng [35], where the delta shock wave with Dirac delta function only in density was found. Besides, Pang [36, 37] dealt with the Riemann problem for system (1.1) with the Chaplyin gas and the generalized Chaplyin gas, and the delta shock wave with Dirac delta function in both the density and the internal energy was revealed. Recently, we solved the Riemann problem to system (1.1) with $f=\beta$ and $p=0$; meanwhile, the influence of the external force on the characteristic curves, contact discontinuities and the delta shock waves was clearly shown. 
Based on the discussions above, the goal of this paper is to consider system (1.1) with a constant external force for the Chaplygin gas in the following form:

$$
\left\{\begin{array}{l}
\rho_{t}+(\rho u)_{x}=0 \\
(\rho u)_{t}+\left(\rho u^{2}-\rho^{-1}\right)_{x}=\beta \rho \\
\left(\rho u^{2} / 2+H\right)_{t}+\left(\left(\rho u^{2} / 2+H-\rho^{-1}\right) u\right)_{x}=\beta \rho u
\end{array}\right.
$$

here $H \geq 0$ denotes the internal energy. The initial data are given as

$$
(\rho, u, H)(0, x)= \begin{cases}\left(\rho_{-}, u_{-}, H_{-}\right), & x<0, \\ \left(\rho_{+}, u_{+}, H_{+}\right), & x>0,\end{cases}
$$

where $\rho_{i}>0, u_{i}, H_{i}>0, i=-,+$, are constants. The generalized Chaplygin gas case will be considered in our future work.

According to (1.2), the physically relevant region of system (1.5) is presented. To solve original Riemann problem (1.5) and (1.6), we write system (1.5) to a conservative one by a change of state variable $v=u-\beta t$ [38]. On the corresponding Riemann problem for the conservative system of (1.5), we construct the solutions when $u_{-}-\rho_{-}^{-1}<u_{+}+\rho_{+}^{-1}$ using the method of analysis in phase space which consists of contact discontinuities. However, when $u_{-}-\rho_{-}^{-1} \geq u_{+}+\rho_{+}^{-1}$, the solutions cannot be obtained in a similar manner, while the occurrence of delta shock wave with Dirac delta function in both density and internal energy is clarified rigorously. In order to describe the delta shock wave, we establish its generalized Rankine-Hugoniot relation and entropy condition, from which its position, speed and weights are calculated. The delta shock wave solution is constructed in this case. Based on these preparations, for the original Riemann problem, making a transformation of state variables, we obtain a unique solution on the physically relevant region when $u_{-}-$ $\rho_{-}^{-1}<u_{+}+\rho_{+}^{-1}$, which is a combination of contact discontinuities. Besides, as $u_{-}-\rho_{-}^{-1} \geq$ $u_{+}+\rho_{+}^{-1}$, we propose both the generalized Rankine-Hugoniot relation and the entropy condition for the delta shock wave, from which we derive explicitly the position, speed and weights of the delta shock wave. Then the delta shock wave solution is obtained on the physically relevant region.

In our study, the delta shock wave with Dirac delta function in both the density and the internal energy occurs in the solution to the compressible perfect fluid with a constant external force for the Chaplygin gas, where the formation of delta shock wave stems from the overlapping of the linearly degenerate characteristic curves. Besides, it is shown that the characteristic curves, the contact discontinuities and the delta shock waves are bent into parabolic shapes under the influence of the constant external force, such that the solutions are no longer self-similar. Moreover, as the constant external force vanishes, the solutions tend to the corresponding ones of the compressible Euler equations for the Chaplygin gas with the same initial data [36].

This paper is organized as follows. In Sect. 2, we rewrite system (1.5) to a conservative system, and investigate the Riemann problem for this conservative system. We also confirm the phenomenon of the delta shock wave with Dirac delta function in both the density and the internal energy. Meanwhile we establish both generalized Rankine-Hugoniot relation and entropy condition for the delta shock wave in the conservative system of (1.5), from which the position, speed and weights of the delta shock wave are shown. The 
solutions to this Rieman problem are constructed. In Sect. 3, the generalized RankineHugoniot relation for the delta shock wave in system (1.5) are derived. By a transformation of the state variables, we obtain the solutions to the Riemann problem (1.5) and (1.6) on the physically relevant region. Finally, a brief conclusion is given in Sect. 4.

\section{Riemann problem for a conservative form of system (1.5)}

Denote by

$$
\aleph=\left\{(\rho, u, H) \mid \rho>0, H \geq \frac{1}{2 \rho}, u \in R\right\}
$$

the physically relevant region for system (1.5), which can be derived by means of (1.2) [36]. With the help of the change of state variable $v=u-\beta t$ introduced by Faccanoni and Mangeney [38], Eq. (1.5) can be rewritten in the conservative form

$$
\left\{\begin{array}{l}
\rho_{t}+(\rho(v+\beta t))_{x}=0 \\
(\rho v)_{t}+\left(\rho v(v+\beta t)-\rho^{-1}\right)_{x}=0 \\
\left(\rho v^{2} / 2+H\right)_{t}+\left(\left(\rho v^{2} / 2+H\right)(v+\beta t)-\rho^{-1} v\right)_{x}=0
\end{array}\right.
$$

Meanwhile, the corresponding initial data are prescribed as

$$
(\rho, v, H)(0, x)= \begin{cases}\left(\rho_{-}, u_{-}, H_{-}\right), & x<0, \\ \left(\rho_{+}, u_{+}, H_{+}\right), & x>0,\end{cases}
$$

and the physically relevant region $\aleph$ in $(\rho, v, H)$-space becomes the region

$$
\aleph^{*}=\left\{(\rho, v, H) \mid \rho>0, H \geq \frac{1}{2 \rho}, v \in R\right\} .
$$

Subsequently, we deal with the Riemann problem (2.2) and (2.3) in detail.

\subsection{Classical wave solutions}

The system (2.2) has three eigenvalues,

$$
\lambda_{1}=v+\beta t-\rho^{-1}, \quad \lambda_{2}=v+\beta t, \quad \lambda_{3}=v+\beta t+\rho^{-1},
$$

with the corresponding right eigenvectors

$$
r_{1}=\left(-\rho^{2}, 1,1-\rho H\right)^{T}, \quad r_{2}=(0,0,1)^{T}, \quad r_{3}=\left(\rho^{2}, 1, \rho H-1\right)^{T},
$$

satisfying $\nabla \lambda_{i} \cdot r_{i}=0, i=1,2,3$. Thus, Eq. (2.2) is a linearly degenerate and strictly hyperbolic system, and the associated waves for them are contact discontinuities.

For bounded discontinuous solutions with a discontinuity $x=x(t)$, the RankineHugoniot condition for (2.2) is shown as

$$
\left\{\begin{array}{l}
-\omega[\rho]+[\rho(v+\beta t)]=0 \\
-\omega[\rho v]+\left[\rho v(v+\beta t)-\rho^{-1}\right]=0 \\
-\omega\left[\rho v^{2} / 2+H\right]+\left[\left(\rho v^{2} / 2+H\right)(v+\beta t)-\rho^{-1} v\right]=0
\end{array}\right.
$$


where $\omega=x^{\prime}(t)$ is the speed of the discontinuity, and $[G]=G_{+}-G_{-}$, with $G_{-}$and $G_{+}$the value of the function $G$ on the left- and right-hand sides of the discontinuity, is the jump of $G$ across the discontinuity. Solving (2.7) yields

$$
\begin{aligned}
& \left\{\begin{array}{l}
\omega_{1}=v_{-}+\beta t-\rho_{-}^{-1}=v_{+}+\beta t-\rho_{+}^{-1}, \\
v_{+}=\rho_{+}^{-1}+v_{-}-\rho_{-}^{-1}, \quad H_{+}=\rho_{+}^{-1} / 2+\rho_{+} \rho_{-}^{-1}\left(H_{-}-\rho_{-}^{-1} / 2\right),
\end{array}\right. \\
& \left\{\begin{array}{l}
\omega_{2}=v_{-}+\beta t=v_{+}+\beta t, \\
\rho_{+}=\rho_{-}, \quad v_{+}=v_{-},
\end{array} H_{+} \neq H_{-},\right. \\
& \begin{cases}\omega_{3}=v_{-}+\beta t+\rho_{-}^{-1}=v_{+}+\beta t+\rho_{+}^{-1}, \\
v_{+}=-\rho_{+}^{-1}+v_{-}+\rho_{-}^{-1}, & H_{+}=\rho_{+}^{-1} / 2+\rho_{+} \rho_{-}^{-1}\left(H_{-}-\rho_{-}^{-1} / 2\right) .\end{cases}
\end{aligned}
$$

Equations (2.8), (2.9) and (2.10) are three kinds of contact discontinuities, denoted by $J_{1}$, $J_{2}$ and $J_{3}$.

On the region $\aleph^{*}$, from point $A\left(\rho_{-}, u_{-}, H_{-}\right)$, we draw the contact discontinuity curves

$$
\begin{array}{ll}
J_{1}\left(\rho_{-}, u_{-}, H_{-}\right): v=u_{-}-\rho_{-}^{-1}+\rho^{-1}, & H=\rho^{-1} / 2+\rho \rho_{-}^{-1}\left(H_{-}-\rho_{-}^{-1} / 2\right), \\
J_{3}\left(\rho_{-}, u_{-}, H_{-}\right): v=u_{-}+\rho_{-}^{-1}-\rho^{-1}, & H=\rho^{-1} / 2+\rho \rho_{-}^{-1}\left(H_{-}-\rho_{-}^{-1} / 2\right),
\end{array}
$$

and a surface

$$
S\left(\rho_{-}, u_{-}, H_{-}\right): v=u_{-}-\rho_{-}^{-1}-\rho^{-1} .
$$

The projection of $J_{1}\left(\rho_{-}, u_{-}, H_{-}\right), J_{3}\left(\rho_{-}, u_{-}, H_{-}\right), S\left(\rho_{-}, u_{-}, H_{-}\right)$onto the $(\rho, v)$-plane has, respectively, two asymptotes $v=u_{-}-\rho_{-}^{-1}$ and $\rho=0, \rho=0$ and $v=u_{-}+\rho_{-}^{-1}, \rho=0$ and $v=u_{-}-\rho_{-}^{-1}$. These projections divide the $(\rho, v)$-plane into five regions $I, I I, I I I, I V$ and $V$, as shown in Fig. 1.

Let us now construct the solutions to the Riemann problem (2.2) and (2.3). On the region $\aleph^{*}$, when the projection of state $\left(\rho_{+}, u_{+}, H_{+}\right)$onto the $(\rho, v)$-plane lies in $I \cup I I \cup I I I \cup I V$, namely, $u_{-}-\rho_{-}^{-1}<u_{+}+\rho_{+}^{-1}$, we draw the contact discontinuity curves $J_{1}\left(\rho_{-}, u_{-}, H_{-}\right)$, and

$$
J_{3}\left(\rho_{+}, u_{+}, H_{+}\right): v=u_{+}+\rho_{+}^{-1}-\rho^{-1}, \quad H=\rho^{-1} / 2+\rho \rho_{+}^{-1}\left(H_{+}-\rho_{+}^{-1} / 2\right) .
$$

The projections of these contact discontinuity curves onto the $(\rho, v)$-plane have a unique intersection $\left(\rho_{\star}, v_{\star}\right)$ determined by

$$
u_{-}-\rho_{-}^{-1}=v_{\star}-\rho_{\star}^{-1}, \quad u_{+}+\rho_{+}^{-1}=v_{\star}+\rho_{\star}^{-1} .
$$

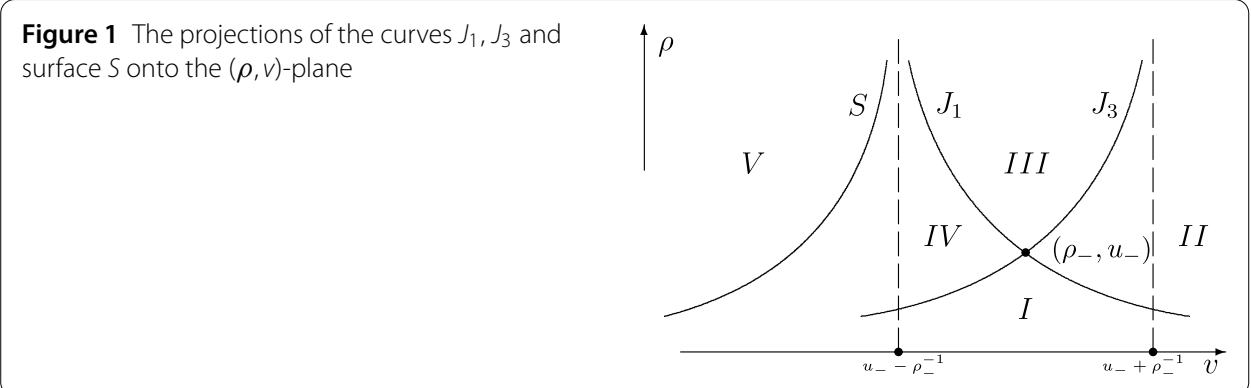


We then draw the contact discontinuity curve

$$
J_{2}\left(\rho_{\star}, v_{\star}, H_{-}\right): \rho=\rho_{\star}, \quad v=v_{\star},
$$

which intersects the contact discontinuity curves $J_{1}\left(\rho_{-}, u_{-}, H_{-}\right)$and $J_{3}\left(\rho_{+}, u_{+}, H_{+}\right)$at the unique points $\left(\rho_{*_{1}}, v_{*_{1}}, H_{*_{1}}\right)$ and $\left(\rho_{*_{2}}, v_{*_{2}}, H_{*_{2}}\right)$. The solution is described by the following theorem.

Theorem 2.1 On the region $\aleph^{*}$, under the condition $u_{+}+\rho_{+}^{-1}>u_{-}-\rho_{-}^{-1}$, the solution to (2.2) and (2.3) can be expressed as

$$
(\rho, v, H)(t, x)= \begin{cases}\left(\rho_{-}, u_{-}, H_{-}\right), & x<x_{1}, \\ \left(\rho_{*_{1}}, v_{*_{1}}, H_{*_{1}}\right), & x_{1} \leq x \leq x_{2} \\ \left(\rho_{*_{2}}, v_{*_{2}}, H_{*_{2}}\right), & x_{2}<x \leq x_{3} \\ \left(\rho_{+}, u_{+}, H_{+}\right), & x>x_{3},\end{cases}
$$

where

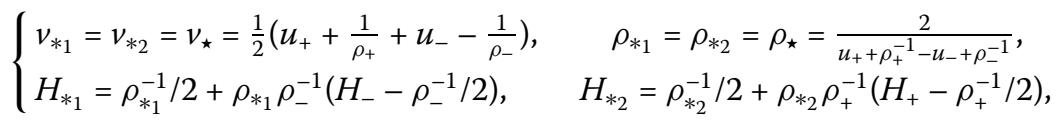

and the contact discontinuities $J_{1}, J_{2}$ and $J_{3}$ are given by $x_{1}(t)=\left(u_{-}-\rho_{-}^{-1}\right) t+\beta t^{2} / 2, x_{2}(t)=$ $v_{\star} t+\beta t^{2} / 2$ and $x_{3}(t)=\left(u_{+}+\rho_{+}^{-1}\right) t+\beta t^{2} / 2$.

\subsection{Delta shock wave solution}

In this subsection, we discuss the case when the projection of the state $\left(\rho_{+}, u_{+}, H_{+}\right)$onto the $(\rho, v)$-plane lies in $V$, namely, $u_{+}+\rho_{+}^{-1} \leq u_{-}-\rho_{-}^{-1}$. The characteristic curves defined by

$$
\frac{\mathrm{d} x_{i}^{ \pm}(t)}{\mathrm{d} t}=\lambda_{i}\left(\rho_{ \pm}, u_{ \pm}\right), \quad i=1,2,3
$$

will overlap in the domain $\Omega=\left\{(t, x) \mid\left(u_{+}+\rho_{+}^{-1}\right) t+\beta t^{2} / 2 \leq x \leq\left(u_{-}-\rho_{-}^{-1}\right) t+\beta t^{2} / 2\right.$, $0 \leq t<+\infty\}$, as illustrated in Fig. 2. Hence, some singularities will happen in $\Omega$, while the singularities cannot be a jump with finite amplitudes.

Figure 2 The domain $\Omega$

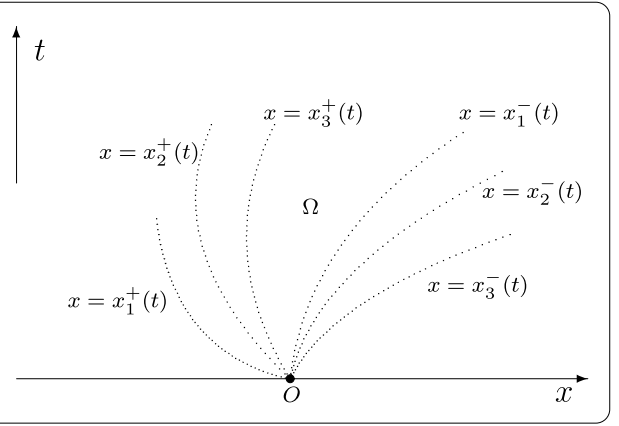


We first study the special case $u_{+}+\rho_{+}^{-1}=u_{-}-\rho_{-}^{-1}$ to show the singularity in $\Omega$. When $\rho_{-}, u_{-}, H_{-}, \rho_{+}, H_{+}$are fixed, $u_{+} \rightarrow u_{-}-\rho_{-}^{-1}-\rho_{+}^{-1}+0$, it gives from (2.16) that

$$
\left\{\begin{array}{l}
v_{*_{1}}=v_{*_{2}} \rightarrow u_{-}-\rho_{-}^{-1}=u_{+}+\rho_{+}^{-1}, \\
\rho_{*_{1}}=\rho_{*_{2}} \rightarrow+\infty, \\
H_{*_{1}} \rightarrow \begin{cases}+\infty, & \text { if } H_{-}>\rho_{-}^{-1} / 2, \\
0, & \text { if } H_{-}=\rho_{-}^{-1} / 2,\end{cases} \\
H_{*_{2}} \rightarrow \begin{cases}+\infty, & \text { if } H_{+}>\rho_{+}^{-1} / 2, \\
0, & \text { if } H_{+}=\rho_{+}^{-1} / 2 .\end{cases}
\end{array}\right.
$$

Therefore the three contact discontinuities $J_{1}, J_{2}$ and $J_{3}$ coincide to form a new singularity. Besides, for any fixed time $t>0$, we deduce that

$$
\begin{aligned}
& \lim _{u_{+} \rightarrow u_{-}-\rho_{-}^{-1}-\rho_{+}^{-1}+0} \int_{\left(u_{-}-\rho_{-}^{-1}\right) t+\beta t^{2} / 2}^{\left(u_{+}+\rho_{+}^{-1}\right) t+\beta t^{2} / 2} \rho \mathrm{d} x \\
& =\lim _{u_{+} \rightarrow u_{-}-\rho_{-}^{-1}-\rho_{+}^{-1}+0} \int_{\left(u_{-}-\rho_{-}^{-1}\right) t+\beta t^{2} / 2}^{\left(u_{+}+\rho_{+}^{-1}\right) t+\beta t^{2} / 2} \rho_{*_{1}} \mathrm{~d} x=2 t, \\
& \quad \lim _{u_{+} \rightarrow u_{-}-\rho_{-}^{-1}-\rho_{+}^{-1}+0} \int_{\left(u_{-}-\rho_{-}^{-1}\right) t+\beta t^{2} / 2}^{\left(u_{+}+\rho_{+}^{-1}\right) t+\beta t^{2} / 2} v \mathrm{~d} x \\
& =\lim _{u_{+} \rightarrow u_{-}-\rho_{-}^{-1}-\rho_{+}^{-1}+0} \int_{\left(u_{-}-\rho_{-}^{-1}\right) t+\beta t^{2} / 2}^{\left(u_{+}+\rho_{+}^{-1}\right) t+\beta t^{2} / 2} \nu_{*_{1}} \mathrm{~d} x=0, \\
& \quad \lim _{u_{+} \rightarrow u_{-}-\rho_{-}^{-1}-\rho_{+}^{-1}+0} \int_{\left(u_{-}-\rho_{-}^{-1}\right) t+\beta t^{2} / 2}^{\left(u_{+}+\rho_{+}^{-1}\right) t+\beta t^{2} / 2} H \mathrm{~d} x \\
& =\lim _{u_{+} \rightarrow u_{-}-\rho_{-}^{-1}-\rho_{+}^{-1}+0}\left(\int_{\left(u_{-}-\rho_{-}^{-1}\right) t+\beta t^{2} / 2}^{v_{*} t+\beta t^{2} / 2} H_{*_{1}} \mathrm{~d} x+\int_{v_{*_{1}} t+\beta t^{2} / 2}^{\left(u_{+}+\rho_{+}^{-1}\right) t+\beta t^{2} / 2} H_{*_{2}} \mathrm{~d} x\right) \\
& =\left(\rho_{-}^{-1}\left(H_{-}-\rho_{-}^{-1} / 2\right)+\rho_{+}^{-1}\left(H_{+}-\rho_{+}^{-1} / 2\right)\right) t \\
& \neq 0, \quad \text { if } H_{-}>\rho_{-}^{-1} / 2 \text { or } H_{+}>\rho_{+}^{-1} / 2 .
\end{aligned}
$$

Hence, if $H_{-}>\rho_{-}^{-1} / 2$ or $H_{+}>\rho_{+}^{-1} / 2$, Eqs. (2.19)-(2.22) show that both $\rho$ and $H$ have the same singularity as a weighed Dirac delta function supported by the curve $x=\left(u_{-}-\rho_{-}^{-1}\right) t+$ $\beta t^{2} / 2$, and that $v$ has a bounded variation. These facts reveal the occurrence of a delta shock wave with a Dirac delta function in the variables $\rho$ and $H$. Moreover, the inequality

$$
u_{+}+\rho_{+}^{-1}+\beta t \leq \sigma(t) \leq u_{-}-\rho_{-}^{-1}+\beta t
$$

holds, where $\sigma(t)$ is the velocity of delta shock wave, which means that all of the six characteristic curves from the initial data on its two sides are incoming.

Therefore, based on the analysis above, the delta shock wave with Dirac delta function in variables $\rho$ and $H$ is suggested in $\Omega$ for the case $u_{+}+\rho_{+}^{-1} \leq u_{-}-\rho_{-}^{-1}$. 
Definition 2.2 ([12]) The two-dimensional weighted Dirac delta function $w(s) \delta_{L}$ supported on a smooth curve $L$ parameterized as $x=x(s), y=y(s)(c \leq s \leq d)$ is defined as

$$
\left\langle w(s) \delta_{L}, \phi(x, y)\right\rangle=\int_{c}^{d} w(s) \phi(x(s), y(s)) \mathrm{d} s
$$

for all the test functions $\phi \in C_{0}^{\infty}\left(R^{2}\right)$.

Definition 2.3 The distribution $(\rho, v, H)$ is a delta shock wave solution of (2.2) and (2.3) in the sense of distributions if there exist a smooth curve $L$ and two functions $w(t), h(t) \in$ $C^{1}(L)$ such that $\rho, v, H$ are of the following form:

$$
\rho=\bar{\rho}(t, x)+w(t) \delta_{L}, \quad v=\bar{v}(t, x), \quad H=\bar{H}(t, x)+h(t) \delta_{L},
$$

and

$$
\left\{\begin{array}{l}
\left\langle\rho, \phi_{t}\right\rangle+\left\langle\rho(v+\beta t), \phi_{x}\right\rangle=0 \\
\left\langle\rho v, \phi_{t}\right\rangle+\left\langle\rho v(v+\beta t)-\rho^{-1}, \phi_{x}\right\rangle=0 \\
\left\langle\rho v^{2} / 2+H, \phi_{t}\right\rangle+\left\langle\left(\rho v^{2} / 2+H\right)(v+\beta t)-\rho^{-1} v, \phi_{x}\right\rangle=0
\end{array}\right.
$$

for all the test functions $\phi \in C_{0}^{\infty}((0,+\infty) \times R)$, where $\bar{\rho}, \bar{v}, \bar{H} \in L^{\infty}([0,+\infty) \times R ; R),\left.v\right|_{L}=$ $v_{\delta}(t)$,

$$
\begin{aligned}
& \langle\rho, \phi\rangle=\int_{0}^{+\infty} \int_{-\infty}^{+\infty} \bar{\rho}(t, x) \phi \mathrm{d} x \mathrm{~d} t+\left\langle w(t) \delta_{L}, \phi\right\rangle, \\
& \langle\rho v, \phi\rangle=\int_{0}^{+\infty} \int_{-\infty}^{+\infty} \bar{\rho} \bar{v}(t, x) \phi \mathrm{d} x \mathrm{~d} t+\left\langle w(t) v_{\delta}(t) \delta_{L}, \phi\right\rangle,
\end{aligned}
$$

and $H$ obeys similar integral identities to the above.

With Definitions 2.2-2.3, we seek the delta shock wave solution to (2.2) and (2.3) in the form

$$
(\rho, v, H)(t, x)= \begin{cases}\left(\rho_{-}, u_{-}, H_{-}\right), & x<x(t) \\ \left(w(t) \delta(x-x(t)), v_{\delta}(t), h(t) \delta(x-x(t))\right), & x=x(t) \\ \left(\rho_{+}, u_{+}, H_{+}\right), & x>x(t)\end{cases}
$$

where $\rho_{i}, u_{i}, H_{i}, i=-,+$ are constants, $\delta$ is the standard Dirac delta function supported on the curve $x=x(t), v_{\delta}(t)$ is the value of the variable $v$ on the curve $x=x(t)$, and $w(t), h(t)$ are the weights of the delta shock wave on the variables $\rho$ and $H$.

Lemma 2.4 Assume that (2.26) satisfies the following ordinary equations:

$$
\left\{\begin{array}{l}
\frac{\mathrm{d} x(t)}{\mathrm{d} t}=\sigma(t)=v_{\delta}(t)+\beta t, \\
\frac{\mathrm{d}(t)}{\mathrm{d} t}=\sigma(t)[\rho]-[\rho(v+\beta t)], \\
\frac{\mathrm{d}\left(w(t) v_{\delta}(t)\right)}{\mathrm{d} t}=\sigma(t)[\rho v]-\left[\rho v(v+\beta t)-\rho^{-1}\right], \\
\frac{\mathrm{d}\left(w(t) v_{\delta}^{2}(t) / 2+h(t)\right)}{\mathrm{d} t}=\sigma(t)\left[\rho v^{2} / 2+H\right]-\left[\left(\rho v^{2} / 2+H\right)(v+\beta t)-\rho^{-1} v\right],
\end{array}\right.
$$

then it is a delta shock wave solution to (2.2) and (2.3) in the sense of distributions. 
Proof Using the first and fourth equalities of (2.27), we have

$$
\begin{aligned}
\left\langle\rho v^{2} / 2\right. & \left.+H, \phi_{t}\right\rangle+\left\langle\left(\rho v^{2} / 2+H\right)(v+\beta t)-\rho^{-1} v, \phi_{x}\right\rangle \\
= & \int_{0}^{+\infty} \int_{-\infty}^{+\infty}\left(\rho v^{2} / 2+H\right) \phi_{t}+\left(\left(\rho v^{2} / 2+H\right)(v+\beta t)-v \rho^{-1}\right) \phi_{x} \mathrm{~d} x \mathrm{~d} t \\
& +\int_{0}^{+\infty}\left(w(t) v_{\delta}^{2}(t) / 2+h(t)\right) \phi_{t}+\left(w(t) v_{\delta}^{2}(t) / 2+h(t)\right)\left(v_{\delta}(t)+\beta t\right) \phi_{x} \mathrm{~d} t \\
= & \int_{0}^{+\infty} \int_{-\infty}^{x(t)}\left(\left(\rho_{-} u_{-}^{2} / 2+H_{-}\right) \phi\right)_{t}+\left(\left(\left(\rho_{-} u_{-}^{2} / 2+H_{-}\right)\left(u_{-}+\beta t\right)-v_{-} \rho_{-}^{-1}\right) \phi\right)_{x} \mathrm{~d} x \mathrm{~d} t \\
& +\int_{0}^{+\infty} \int_{x(t)}^{+\infty}\left(\left(\rho_{+} u_{+}^{2} / 2+H_{+}\right) \phi\right)_{t}+\left(\left(\left(\rho_{+} u_{+}^{2} / 2+H_{+}\right)\left(u_{+}+\beta t\right)-v_{+} \rho_{+}^{-1}\right) \phi\right)_{x} \mathrm{~d} x \mathrm{~d} t \\
& +\int_{0}^{+\infty}\left(w(t) v_{\delta}^{2}(t) / 2+h(t)\right)\left(\phi_{t}+\left(v_{\delta}(t)+\beta t\right) \phi_{x}\right) \mathrm{d} t \\
= & -\int_{0}^{+\infty}-\left(\left(\rho_{-} u_{-}^{2} / 2+H_{-}\right)\left(u_{-}+\beta t\right)-v_{-} \rho_{-}^{-1}\right) \phi \mathrm{d} t+\left(\rho_{-} u_{-}^{2} / 2+H_{-}\right) \phi \mathrm{d} x \\
& +\int_{0}^{+\infty}-\left(\left(\rho_{+} u_{+}^{2} / 2+H_{+}\right)\left(u_{+}+\beta t\right)-v_{+} \rho_{+}^{-1}\right) \phi \mathrm{d} t+\left(\rho_{+} u_{+}^{2} / 2+H_{+}\right) \phi \mathrm{d} x \\
& -\int_{0}^{+\infty} \phi \frac{\mathrm{d}\left(w(t) v_{\delta}^{2}(t) / 2+h(t)\right)}{\mathrm{d} t} \mathrm{~d} t \\
= & \int_{0}^{+\infty} \phi\left(\sigma(t)\left[\rho v^{2} / 2+H\right]-\left[\left(\rho v^{2} / 2+H\right)(v+\beta t)-v \rho^{-1}\right]\right. \\
& -\frac{\mathrm{d}\left(w(t) v_{\delta}^{2}(t) / 2+h(t)\right)}{\mathrm{d} t} \mathrm{~d} t \\
= & 0
\end{aligned}
$$

for any test functions $\phi \in C_{0}^{\infty}((0,+\infty) \times R)$, which implies the third equality of $(2.25)$. The others can be proved similarly. The proof is complete.

In addition, to ensure the uniqueness of delta shock wave solution, the curve $x=x(t)$ with $x^{\prime}(t)=\sigma(t)$ satisfies the following inequality:

$$
u_{+}+\rho_{+}^{-1}+\beta t \leq \sigma(t) \leq u_{-}-\rho_{-}^{-1}+\beta t
$$

which means that all of the characteristic curves from the initial data on both sides of $x=x(t)$ are incoming.

Definition 2.5 A discontinuity satisfying the system of equations (2.27) and the inequality (2.29) is called a delta shock wave, denoted by $\delta$.

Remark 2.6 The system of equations (2.27) is called the generalized Rankine-Hugoniot relation of the delta shock wave. It describes the location, speed, weights as well as the assignment of $v$ on the delta shock wave. Besides, the inequality (2.29) is called the entropy condition of the delta shock wave. 
In what follows, we solve the Riemann problem (2.2) and (2.3) when $u_{+}+\rho_{+}^{-1} \leq u_{-}-\rho_{-}^{-1}$ using (2.27) and (2.29). At this moment, this problem is reduced to an initial value problem for (2.27) with initial conditions

$$
x(0)=0, \quad v_{\delta}(0)=0, \quad w(0)=0, \quad h(0)=0 .
$$

We have the following result.

Theorem 2.7 On the region $\aleph^{*}$, under the condition $u_{+}+\rho_{+}^{-1} \leq u_{-}-\rho_{-}^{-1}$, if $H_{-}>\rho_{-}^{-1} / 2$ or $H_{+}>\rho_{+}^{-1} / 2$, then the Riemann problem (2.2) and (2.3) admits uniquely a delta shock wave solution defined in Definition 2.3,

$$
(\rho, v, H)(t, x)= \begin{cases}\left(\rho_{-}, u_{-}, H_{-}\right), & x<x(t) \\ \left(w(t) \delta(x-x(t)), v_{\delta}(t), h(t) \delta(x-x(t))\right), & x=x(t) \\ \left(\rho_{+}, u_{+}, H_{+}\right), & x>x(t)\end{cases}
$$

where

$$
\left\{\begin{aligned}
& v_{\delta}(t)= \begin{cases}\left(u_{+}+u_{-}\right) / 2, & \text { if } \rho_{-}=\rho_{+}, \\
\frac{\rho_{+} u_{+}-\rho_{-} u_{-}+\sqrt{\rho_{+} \rho_{-}\left(\left(u_{-}-u_{+}\right)^{2}-\left(\rho_{-}^{-1}-\rho_{+}^{-1}\right)^{2}\right)}}{\rho_{+}-\rho_{-}}, & \text {if } \rho_{-} \neq \rho_{+}\end{cases} \\
& x(t)=v_{\delta}(t) t+\beta t^{2} / 2, \\
& w(t)=\left(\left(\rho_{+}-\rho_{-}\right) v_{\delta}(t)-\rho_{+} u_{+}+\rho_{-} u_{-}\right) t, \\
& h(t)=\left(\left(u_{-}-v_{\delta}(t)\right)\left(\rho_{-}\left(u_{-}-v_{\delta}(t)\right)^{2} / 2+H_{-}-\rho_{-}^{-1}\right)\right. \\
&\left.+\left(v_{\delta}(t)-u_{+}\right)\left(\rho_{+}\left(v_{\delta}(t)-u_{+}\right)^{2} / 2+H_{+}-\rho_{+}^{-1}\right)\right) t .
\end{aligned}\right.
$$

Proof We deduce from (2.27) and (2.30) that

$$
\left\{\begin{array}{l}
w(t)=[\rho] \int_{0}^{t} v_{\delta}(s) \mathrm{d} s-[\rho v] t, w(t) v_{\delta}(t)=[\rho v] \int_{0}^{t} v_{\delta}(s) \mathrm{d} s-\left[\rho v^{2}-\rho^{-1}\right] t \\
w(t) v_{\delta}^{2}(t) / 2+h(t)=\left[\rho v^{2} / 2+H\right] \int_{0}^{t} v_{\delta}(s) \mathrm{d} s-\left[\left(\rho v^{2} / 2+H-\rho^{-1}\right) v\right] t
\end{array}\right.
$$

Substituting the first equality of (2.33) into the second one leads to

$$
[\rho] v_{\delta}(t) \int_{0}^{t} v_{\delta}(s) \mathrm{d} s-[\rho v]\left(v_{\delta}(t) t+\int_{0}^{t} v_{\delta}(s) \mathrm{d} s\right)+\left[\rho v^{2}-\rho^{-1}\right] t=0
$$

that is,

$$
[\rho]\left(\int_{0}^{t} v_{\delta}(s) \mathrm{d} s\right)^{2}-2[\rho v] t \int_{0}^{t} v_{\delta}(s) \mathrm{d} s+\left[\rho v^{2}-\rho^{-1}\right] t^{2}=0
$$

When $[\rho]=\rho_{+}-\rho_{-}=0$, it is easy to calculate that

$$
\left\{\begin{array}{l}
x(t)=\left(u_{-}+u_{+}\right) t / 2+\beta t^{2} / 2, \quad v_{\delta}(t)=\left(u_{-}+u_{+}\right) / 2, \\
w(t)=\rho_{-}\left(u_{-}-u_{+}\right) t, \quad h(t)=\left(\frac{\rho_{-}\left(u_{-}-u_{+}\right)^{2}}{8}+\frac{H_{-}+H_{+}}{2}-\frac{1}{\rho_{-}}\right)\left(u_{-}-u_{+}\right) t,
\end{array}\right.
$$

which satisfies entropy condition (2.29). 
When $[\rho]=\rho_{+}-\rho_{-} \neq 0, u_{+}+\rho_{+}^{-1} \leq u_{-}-\rho_{-}^{-1}$ implies that for (2.34) there exist two solutions,

$$
\int_{0}^{t} v_{\delta}(s) \mathrm{d} s=\frac{1}{\rho_{+}-\rho_{-}}\left(\rho_{+} u_{+}-\rho_{-} u_{-} \pm \sqrt{\rho_{+} \rho_{-}\left(\left(u_{-}-u_{+}\right)^{2}-\left(\rho_{-}^{-1}-\rho_{+}^{-1}\right)^{2}\right)}\right) t
$$

which yields

$$
v_{\delta_{ \pm}}(t)=\frac{1}{\rho_{+}-\rho_{-}}\left(\rho_{+} u_{+}-\rho_{-} u_{-} \pm \sqrt{\rho_{+} \rho_{-}\left(\left(u_{-}-u_{+}\right)^{2}-\left(\rho_{-}^{-1}-\rho_{+}^{-1}\right)^{2}\right)}\right) .
$$

Using entropy condition (2.29), one can choose from (2.36) that

$$
v_{\delta}(t)=\frac{1}{\rho_{+}-\rho_{-}}\left(\rho_{+} u_{+}-\rho_{-} u_{-}+\sqrt{\rho_{+} \rho_{-}\left(\left(u_{-}-u_{+}\right)^{2}-\left(\rho_{-}^{-1}-\rho_{+}^{-1}\right)^{2}\right)}\right) .
$$

Thus we obtain from $(2.33)$

$$
\left\{\begin{aligned}
x(t)= & v_{\delta}(t) t+\beta t^{2} / 2, \quad w(t)=\left(\left(\rho_{+}-\rho_{-}\right) v_{\delta}(t)-\rho_{+} u_{+}+\rho_{-} u_{-}\right) t \\
v_{\delta}(t)= & \frac{1}{\rho_{+}-\rho_{-}}\left(\rho_{+} u_{+}-\rho_{-} u_{-}+\sqrt{\rho_{+} \rho_{-}\left(\left(u_{-}-u_{+}\right)^{2}-\left(\rho_{-}^{-1}-\rho_{+}^{-1}\right)^{2}\right)}\right) \\
h(t)= & \left(\left(u_{-}-v_{\delta}(t)\right)\left(\rho_{-}\left(u_{-}-v_{\delta}(t)\right)^{2} / 2+H_{-}-\rho_{-}^{-1}\right)\right. \\
& \left.+\left(v_{\delta}(t)-u_{+}\right)\left(\rho_{+}\left(v_{\delta}(t)-u_{+}\right)^{2} / 2+H_{+}-\rho_{+}^{-1}\right)\right) t
\end{aligned}\right.
$$

The proof is complete.

Remark 2.8 On the region $\aleph^{*}$, under the condition $u_{+}+\rho_{+}^{-1} \leq u_{-}-\rho_{-}^{-1}$, if $H_{-}=\rho_{-}^{-1} / 2$ and $H_{+}=\rho_{+}^{-1} / 2$, the (2.19)-(2.22) imply that only the variable $\rho$ contains Dirac delta function supported on the curve $x=\left(u_{-}-\rho_{-}^{-1}\right) t+\beta t^{2} / 2$, and that both $v$ and $H$ have a bounded variation. In this special case, one can prove similarly that for the Riemann problem (2.2) and (2.3) there exists uniquely a delta shock wave solution defined in Definition 2.3, in which only the density $\rho$ contains a Dirac delta function.

\section{Riemann problem for system (1.5)}

In this section, we address the original Riemann problem (1.5) and (1.6) based on the preparation in the last section. On the physically relevant region $\aleph$, when $u_{-}-\rho_{-}^{-1}<$ $u_{+}+\rho_{+}^{-1}$, we obtain the solution to (1.5) and (1.6) directly from the corresponding ones to (2.2) and (2.3) by the transformation of the state variables $(\rho, u, H)(t, x)=(\rho, v+\beta t, H)(t, x)$ where the positions of the contact discontinuities remain unchanged, as shown in Fig. 3 . The solution is presented as follows.

Theorem 3.1 On the physically relevant region $\aleph$, under the condition $u_{+}+\rho_{+}^{-1}>u_{-}-\rho_{-}^{-1}$, the solution to (1.5) and (1.6) is described as

$$
(\rho, u, H)(t, x)= \begin{cases}\left(\rho_{-}, u_{-}+\beta t, H_{-}\right), & x<x_{1} \\ \left(\rho_{*_{1}}, v_{*_{1}}+\beta t, H_{*_{1}}\right), & x_{1} \leq x \leq x_{2} \\ \left(\rho_{*_{2}}, v_{*_{2}}+\beta t, H_{*_{2}}\right), & x_{2}<x \leq x_{3} \\ \left(\rho_{+}, u_{+}+\beta t, H_{+}\right), & x>x_{3}\end{cases}
$$




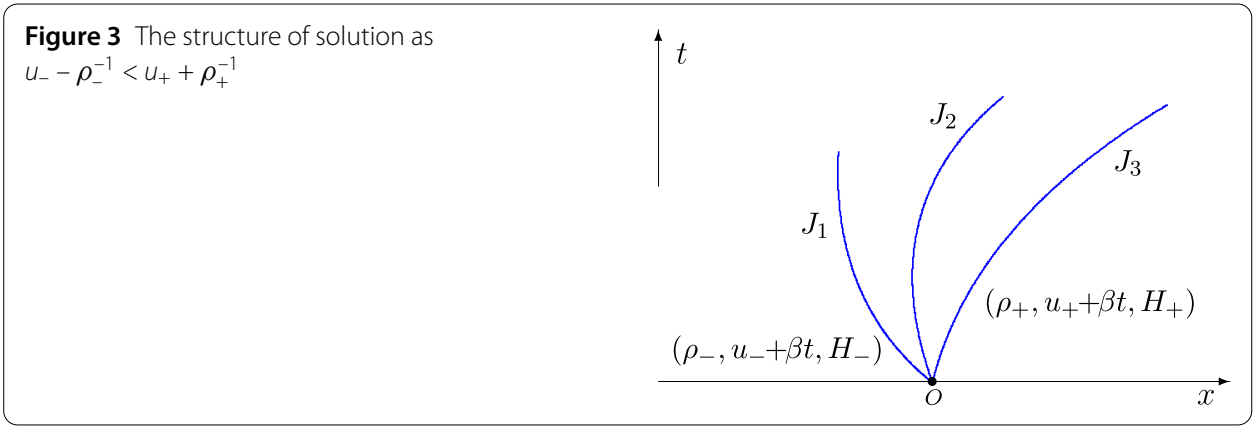

where the $\rho_{*_{1}}, v_{*_{1}}, H_{*_{1}}, \rho_{*_{2}}, v_{*_{2}}, H_{*_{2}}$ are presented in (2.17), and the contact discontinuities $J_{1}, J_{2}$ and $J_{3}$ are given by $x_{1}(t)=\left(u_{-}-\rho_{-}^{-1}\right) t+\beta t^{2} / 2, x_{2}(t)=v_{\star} t+\beta t^{2} / 2$ and $x_{3}(t)=$ $\left(u_{+}+\rho_{+}^{-1}\right) t+\beta t^{2} / 2$.

When $u_{+}+\rho_{+}^{-1} \leq u_{-}-\rho_{-}^{-1}$, one can define a delta shock wave solution to (1.5) and (1.6) as follows.

Definition 3.2 The distribution $(\rho, u, H)$ is a delta shock wave solution of (1.5) and (1.6) in the sense of distributions if there exist a smooth curve $L$ and two functions $w(t), h(t) \in$ $C^{1}(L)$ such that $\rho, u, H$ are of the following form:

$$
\rho=\bar{\rho}(t, x)+w(t) \delta_{L}, \quad u=\bar{u}(t, x), \quad H=\bar{H}(t, x)+h(t) \delta_{L},
$$

and

$$
\left\{\begin{array}{l}
\left\langle\rho, \phi_{t}\right\rangle+\left\langle\rho u, \phi_{x}\right\rangle=0 \\
\left\langle\rho u, \phi_{t}\right\rangle+\left\langle\rho u^{2}-\rho^{-1}, \phi_{x}\right\rangle+\langle\beta \rho, \phi\rangle=0 \\
\left\langle\rho u^{2} / 2+H, \phi_{t}\right\rangle+\left\langle\left(\rho u^{2} / 2+H-\rho^{-1}\right) u, \phi_{x}\right\rangle+\langle\beta \rho u, \phi\rangle=0
\end{array}\right.
$$

for all the test functions $\phi \in C_{0}^{\infty}((0,+\infty) \times R)$, where $\bar{\rho}, \bar{u}, \bar{H} \in L^{\infty}([0,+\infty) \times R ; R),\left.u\right|_{L}=$ $u_{\delta}(t)$

$$
\begin{aligned}
& \langle\rho, \phi\rangle=\int_{0}^{+\infty} \int_{-\infty}^{+\infty} \bar{\rho}(t, x) \phi \mathrm{d} x \mathrm{~d} t+\left\langle w(t) \delta_{L}, \phi\right\rangle \\
& \langle\rho u, \phi\rangle=\int_{0}^{+\infty} \int_{-\infty}^{+\infty} \bar{\rho} \bar{u}(t, x) \phi \mathrm{d} x \mathrm{~d} t+\left\langle w(t) u_{\delta}(t) \delta_{L}, \phi\right\rangle
\end{aligned}
$$

and $H$ obeys similar integral identities to the above.

According to Definition 3.2, we propose the delta shock wave solution to (1.5) and (1.6) in the form

$$
(\rho, u, H)(t, x)= \begin{cases}\left(\rho_{-}, u_{-}+\beta t, H_{-}\right), & x<x(t), \\ \left(w(t) \delta(x-x(t)), u_{\delta}(t), h(t) \delta(x-x(t))\right), & x=x(t), \\ \left(\rho_{+}, u_{+}+\beta t, H_{+}\right), & x>x(t),\end{cases}
$$

where $\left(\rho_{i}, u_{i}, H_{i}\right), i=-,+$ are constants, $\delta$ is the standard Dirac delta function supported on the curve $x=x(t), u_{\delta}(t)$ is the value of the variable $u$ on the curve $x=x(t)$, and $w(t), h(t)$ are the weights of the delta shock wave on the variables $\rho, H$. 
Lemma 3.3 If Eq. (3.4) satisfies the following generalized Rankine-Hugoniot relation:

$$
\left\{\begin{array}{l}
\frac{\mathrm{d} x(t)}{\mathrm{d} t}=\sigma(t)=u_{\delta}(t) \\
\frac{\mathrm{d} w(t)}{\mathrm{d} t}=\sigma(t)[\rho]-[\rho u] \\
\frac{\mathrm{d}\left(w(t) u_{\delta}(t)\right)}{\mathrm{d}(t)}=\sigma(t)[\rho u]-\left[\rho u^{2}-\rho^{-1}\right]+\beta w(t) \\
\frac{\mathrm{d}\left(w(t) u_{\delta}^{2}(t) / 2+h(t)\right)}{\mathrm{d} t}=\sigma(t)\left[\rho u^{2} / 2+H\right]-\left[\left(\rho u^{2} / 2+H-\rho^{-1}\right) u\right]+\beta w(t) u_{\delta}(t)
\end{array}\right.
$$

then it is a delta shock wave solution to (1.5) and (1.6) in the sense of distributions.

Proof Under the conditions (3.5), we deduce that

$$
\begin{aligned}
\left\langle\rho u^{2} / 2\right. & \left.+H, \phi_{t}\right\rangle+\left\langle\left(\rho u^{2} / 2+H-\rho^{-1}\right) u, \phi_{x}\right\rangle+\langle\beta \rho u, \phi\rangle \\
= & \int_{0}^{+\infty} \int_{-\infty}^{+\infty}\left(\rho u^{2} / 2+H\right) \phi_{t}+\left(\rho u^{2} / 2+H-\rho^{-1}\right) u \phi_{x}+\beta \rho u \phi \mathrm{d} x \mathrm{~d} t \\
& +\int_{0}^{+\infty}\left(w(t) u_{\delta}^{2}(t) / 2+h(t)\right) \phi_{t}+\left(w(t) u_{\delta}^{2}(t) / 2+h(t)\right) u_{\delta}(t) \phi_{x}+\beta w(t) u_{\delta}(t) \phi \mathrm{d} t \\
= & \int_{0}^{+\infty} \int_{-\infty}^{x(t)}\left(\left(\rho_{-}\left(u_{-}+\beta t\right)^{2} / 2+H_{-}-\rho_{-}^{-1}\right)\left(u_{-}+\beta t\right) \phi\right)_{x} \\
& +\left(\left(\rho_{-}\left(u_{-}+\beta t\right)^{2} / 2+H_{-}\right) \phi\right)_{t} \mathrm{~d} x \mathrm{~d} t \\
& +\int_{0}^{+\infty} \int_{x(t)}^{+\infty}\left(\left(\rho_{+}\left(u_{+}+\beta t\right)^{2} / 2+H_{+}\right) \phi\right)_{t} \\
& +\left(\left(\rho_{+}\left(u_{+}+\beta t\right)^{2} / 2+H_{+}-\rho_{+}^{-1}\right)\left(u_{+}+\beta t\right) \phi\right)_{x} \mathrm{~d} x \mathrm{~d} t \\
& +\int_{0}^{+\infty}\left(w(t) u_{\delta}^{2}(t) / 2+h(t)\right)\left(\phi_{t}+u_{\delta}(t) \phi_{x}\right)+\beta w(t) u_{\delta}(t) \phi \mathrm{d} t \\
= & -\int_{0}^{+\infty}-\left(\left(\rho_{-}\left(u_{-}+\beta t\right)^{2} / 2+H_{-}-\rho_{-}^{-1}\right)\left(u_{-}+\beta t\right) \phi\right) \\
& +\left(\rho_{-}\left(u_{-}+\beta t\right)^{2} / 2+H_{-}\right) \phi \sigma(t) \mathrm{d} t \\
& +\int_{0}^{+\infty}-\left(\left(\rho_{+}\left(u_{+}+\beta t\right)^{2} / 2+H_{+}-\rho_{+}^{-1}\right)\left(u_{+}+\beta t\right) \phi\right) \\
& +\left(\rho_{+}\left(u_{+}+\beta t\right)^{2} / 2+H_{+}\right) \phi \sigma(t) \mathrm{d} t \\
& +\int_{0}^{+\infty} \beta w(t) u_{\delta}(t) \phi-\phi \frac{\mathrm{d}\left(w(t) u_{\delta}^{2}(t) / 2+h(t)\right)}{\mathrm{d} t} \mathrm{~d} t \\
= & \int_{0}^{+\infty} \phi\left(\sigma(t)\left[\rho u^{2} / 2+H\right]-\left[\left(\rho u^{2} / 2+H-\rho^{-1}\right) u\right]\right. \\
& \left.+\beta(t)-\frac{\mathrm{d}\left(w(t) u_{\delta}^{2}(t) / 2+h(t)\right)}{\mathrm{d} t}\right) \mathrm{d} t \\
& \\
&
\end{aligned}
$$

for any test functions $\phi \in C_{0}^{\infty}((0,+\infty) \times R)$, which yields the third equality of (3.3). The others can be proved in a similar manner. The proof is finished. 


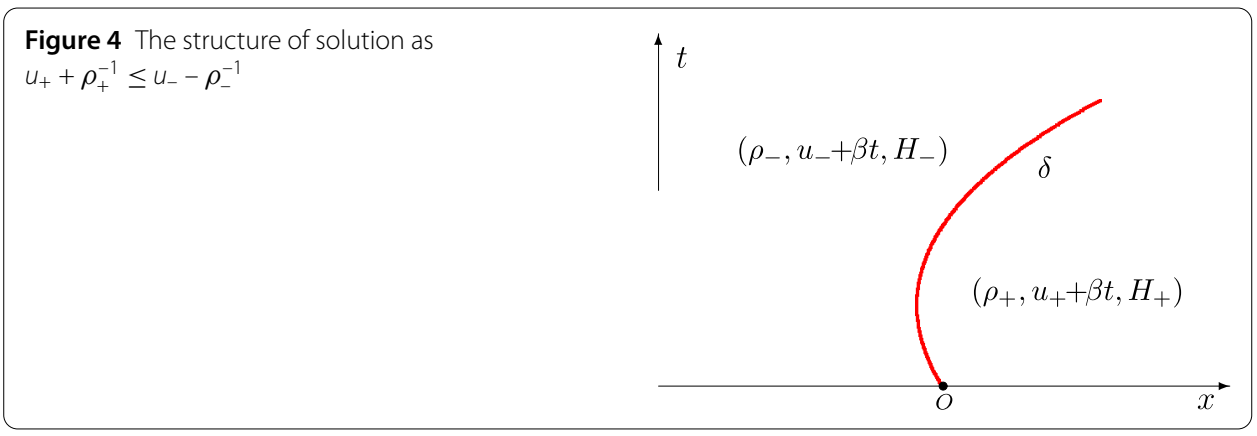

In addition, to guarantee the uniqueness of delta shock wave solution, the curve $x=x(t)$ with $\sigma(t)=x^{\prime}(t)$ satisfies the following entropy condition:

$$
u_{+}+\rho_{+}^{-1}+\beta t \leq \sigma(t) \leq u_{-}-\rho_{-}^{-1}+\beta t
$$

which means that all of the characteristic curves from the initial data on both sides of the curve $x=x(t)$ are incoming.

Subsequently, we solve Riemann problem (1.5) and (1.6) when $u_{+}+\rho_{+}^{-1}<u_{-}-\rho_{-}^{-1}$ using (3.5) and (3.7). At this moment, we need to handle an initial value problem for (3.5) with initial conditions

$$
x(0)=0, \quad u_{\delta}(0)=0, \quad w(0)=0, \quad h(0)=0 .
$$

The solution is described by the following theorem, as shown in Fig. 4.

Theorem 3.4 On the physically relevant region $\aleph$, under the condition $u_{+}+\rho_{+}^{-1} \leq u_{-}-$ $\rho_{-}^{-1}$, if $H_{-}>\rho_{-}^{-1} / 2$ or $H_{+}>\rho_{+}^{-1} / 2$, then for the Riemann problem (1.5) and (1.6) there exists uniquely a delta shock wave solution defined in Definition 3.2,

$$
(\rho, u, H)(t, x)= \begin{cases}\left(\rho_{-}, u_{-}+\beta t, H_{-}\right), & x<x(t), \\ \left(w(t) \delta(x-x(t)), v_{\delta}(t)+\beta t, h(t) \delta(x-x(t))\right), & x=x(t), \\ \left(\rho_{+}, u_{+}+\beta t, H_{+}\right), & x>x(t)\end{cases}
$$

where $x(t), v_{\delta}(t), w(t)$ and $h(t)$ are given by $(2.32)$.

Proof One derives from (3.5) and (3.8) that

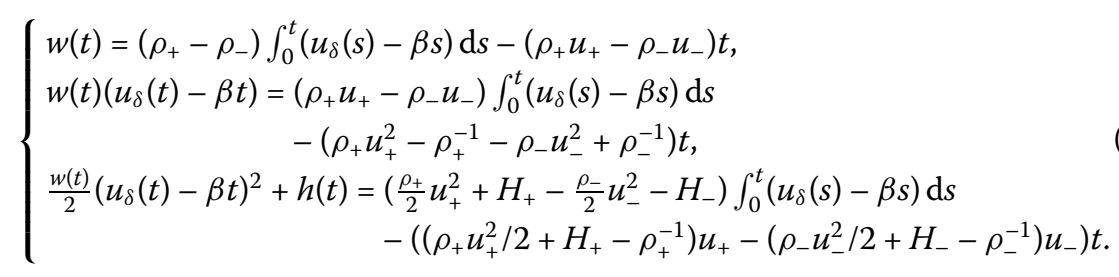

Substituting the first equality of (3.10) into the second one yields

$$
\begin{gathered}
\left(\rho_{+}-\rho_{-}\right)\left(u_{\delta}(t)-\beta t\right) \int_{0}^{t}\left(u_{\delta}(s)-\beta s\right) \mathrm{d} s+\left(\rho_{+} u_{+}^{2}-\rho_{+}^{-1}-\rho_{-} u_{-}^{2}+\rho_{-}^{-1}\right) t \\
-\left(\rho_{+} u_{+}-\rho_{-} u_{-}\right)\left(\left(u_{\delta}(t)-\beta t\right) t+\int_{0}^{t}\left(u_{\delta}(s)-\beta s\right) \mathrm{d} s\right)=0,
\end{gathered}
$$


that is,

$$
\begin{aligned}
& \left(\rho_{+}-\rho_{-}\right)\left(\int_{0}^{t}\left(u_{\delta}(s)-\beta s\right) \mathrm{d} s\right)^{2}-2\left(\rho_{+} u_{+}-\rho_{-} u_{-}\right) t \int_{0}^{t}\left(u_{\delta}(s)-\beta s\right) \mathrm{d} s \\
& +\left(\rho_{+} u_{+}^{2}-\rho_{+}^{-1}-\rho_{-} u_{-}^{2}+\rho_{-}^{-1}\right) t^{2}=0 .
\end{aligned}
$$

If $[\rho]=\rho_{+}-\rho_{-}=0$, one shows that

$$
u_{\delta}(t)=\left(u_{-}+u_{+}\right) / 2+\beta t=v_{\delta}(t)+\beta t
$$

Thus, a simple calculation from (3.10) shows that

$$
\left\{\begin{array}{l}
x(t)=v_{\delta}(t) t+\beta t^{2} / 2, u_{\delta}(t)=v_{\delta}(t)+\beta t, \\
w(t)=\rho_{-}\left(u_{-}-u_{+}\right) t, \quad h(t)=\left(\frac{\rho_{-}\left(u_{-}-u_{+}\right)^{2}}{8}+\frac{H_{-}+H_{+}}{2}-\frac{1}{\rho_{-}}\right)\left(u_{-}-u_{+}\right) t,
\end{array}\right.
$$

which satisfies the entropy condition (3.7).

If $[\rho]=\rho_{+}-\rho_{-} \neq 0$, the inequality $u_{+}+\rho_{+}^{-1} \leq u_{-}-\rho_{-}^{-1}$ implies that Eq. (3.11) has two solutions

$$
\int_{0}^{t}\left(u_{\delta}(s)-\beta s\right) \mathrm{d} s=\frac{1}{\rho_{+}-\rho_{-}}\left(\rho_{+} u_{+}-\rho_{-} u_{-} \pm \sqrt{\rho_{+} \rho_{-}\left(\left(u_{-}-u_{+}\right)^{2}-\left(\rho_{-}^{-1}-\rho_{+}^{-1}\right)^{2}\right)}\right) t
$$

that is,

$$
u_{\delta_{ \pm}}(t)=\frac{1}{\rho_{+}-\rho_{-}}\left(\rho_{+} u_{+}-\rho_{-} u_{-} \pm \sqrt{\rho_{+} \rho_{-}\left(\left(u_{-}-u_{+}\right)^{2}-\left(\rho_{-}^{-1}-\rho_{+}^{-1}\right)^{2}\right)}\right)+\beta t .
$$

With the entropy condition (3.7), one can check from (3.13) that

$$
\begin{aligned}
& u_{\delta}(t)=\frac{1}{\rho_{+}-\rho_{-}}\left(\rho_{+} u_{+}-\rho_{-} u_{-}+\sqrt{\rho_{+} \rho_{-}\left(\left(u_{-}-u_{+}\right)^{2}-\left(\rho_{-}^{-1}-\rho_{+}^{-1}\right)^{2}\right)}\right)+\beta t \\
& =v_{\delta}(t)+\beta t \text {. }
\end{aligned}
$$

Therefore, one can infer from (3.10) that

$$
\left\{\begin{aligned}
x(t)= & v_{\delta}(t) t+\beta t^{2} / 2, \quad u_{\delta}(t)=v_{\delta}(t)+\beta t \\
w(t)= & \left(\left(\rho_{+}-\rho_{-}\right) v_{\delta}(t)-\rho_{+} u_{+}+\rho_{-} u_{-}\right) t \\
h(t)= & \left(\left(u_{-}-v_{\delta}(t)\right)\left(\rho_{-}\left(u_{-}-v_{\delta}(t)\right)^{2} / 2+H_{-}-\rho_{-}^{-1}\right)\right. \\
& \left.+\left(v_{\delta}(t)-u_{+}\right)\left(\rho_{+}\left(v_{\delta}(t)-u_{+}\right)^{2} / 2+H_{+}-\rho_{+}^{-1}\right)\right) t .
\end{aligned}\right.
$$

We have finished the proof of the theorem.

Remark 3.5 It is observed that, under the influence of a constant external force, the characteristic curves, the contact discontinuities and the delta shock waves are bent into parabolic shapes, such that the solutions (3.1) and (3.9) are no longer self-similar any more. It is also shown that, when the constant external force vanishes, the solutions converge to the corresponding ones of the compressible Euler equations for the Chaplygin gas with the same initial data [36]. 


\section{Conclusion}

We solve completely the Riemann problem for a compressible perfect fluid with a constant external force for the Chaplygin gas. The delta shock wave with Dirac delta function in both density and internal energy arises in the solutions for certain initial data, where the formation of the delta shock wave stems from the overlapping of the linearly degenerate characteristic curves. It is also noticed that the solutions are no longer self-similar anymore due to the influence of the constant external force. Moreover, this work provides important ideas and techniques for handling the Riemann problem for system (1.1) with the generalized Chaplygin gas.

\section{Acknowledgements}

The authors would like to thank the referees for their valuable suggestions which have significantly improved the paper.

\section{Funding}

This work is partially supported by the National Natural Science Foundation of China (11661015), the Science and Technology Foundation of Guizhou Province (J[2015]2026) and the Project of High Level Creative Talents in Guizhou Province.

Availability of data and materials

Not applicable.

\section{Competing interests}

The authors declare that they have no competing interests.

Authors' contributions

All authors contributed equally and significantly in writing this article. All authors read and approved the final manuscript.

\section{Author details}

${ }^{1}$ School of Mathematics and Statistics, Guizhou University of Finance and Economics, Guiyang, China. ${ }^{2}$ Department of Mathematics and Information Science, Tangshan Normal University, Tangshan, China.

\section{Publisher's Note}

Springer Nature remains neutral with regard to jurisdictional claims in published maps and institutional affiliations.

\section{Received: 1 March 2018 Accepted: 25 May 2018 Published online: 01 June 2018}

\section{References}

1. Lions, P.L.: Mathematical Topics in Fluid Mechanics: Volume 1: Incompressible Models. Oxford University Press, New York (1996)

2. Lions, P.L.: Mathematical Topics in Fluid Mechanics: Volume 2: Compressible Models. Oxford University Press, New York (1998)

3. Chaplygin, S.: On gas jets. Sci. Mem. Moscow Univ. Math. Phys. 21, 1-121 (1902)

4. Tsien, H.S.: Two-dimensional subsonic flow of compressible fluids. J. Aeronaut. Sci. 6, 399-407 (1939)

5. von Karman, T.: Compressibility effects in aerodynamics. J. Aeronaut. Sci. 8, 337-365 (1941)

6. Bilic, N., Tupper, G.B., Viollier, R.D.: Dark matter, dark energy and the Chaplygin gas. arXiv:astro-ph/0207423

7. Gorini, V., Kamenshchik, A., Moschella, U., Pasquier, V.: The Chaplygin gas as a model for dark energy. arXiv:gr-qc/0403062

8. Setare, M.R. Holographic Chaplygin gas model. Phys. Lett. B 648, 329-332 (2007)

9. Brenier, Y: Solutions with concentration to the Riemann problem for one-dimensional Chaplygin gas equations. J. Math. Fluid Mech. 7, S326-S331 (2005)

10. Guo, L., Sheng, W., Zhang, T.: The two-dimensional Riemann problem for isentropic Chaplygin gas dynamic system. Commun. Pure Appl. Anal. 9, 431-458 (2010)

11. Tan, D., Zhang, T., Zheng, Y.: Delta-shock waves as limits of vanishing viscosity for hyperbolic systems of conservation laws. J. Differ. Equ. 112, 1-32 (1994)

12. Li, J., Zhang, T., Yang, S.L.: The Two-dimensional Riemann Problem in Gas Dynamics. Longman, London (1998)

13. Sheng, W.C., Zhang, T.: The Riemann problem for the transportation equations in gas dynamics. Mem. Am. Math. Soc $137,1-77(1999)$

14. Yang, H.: Riemann problems for a class of coupled hyperbolic systems of conservation laws. J. Differ. Equ. 159, 447-484 (1999)

15. Chen, G., Liu, H.: Formation of $\delta$-shocks and vacuum states in the vanishing pressure limit of solutions to the Euler equations for nonisentropic fluids. SIAM J. Math. Anal. 34, 925-938 (2003)

16. Danilov, V.G., Shelkovich, V.M.: Dynamics of propagation and interaction of $\delta$-shock waves in conservation law systems. J. Differ. Equ. 211, 333-381 (2005)

17. Wang, G.: The Riemann problem for one dimensional generalized Chaplygin gas dynamics. J. Math. Anal. Appl. 403 434-450 (2013) 
18. Sheng, W., Wang, G., Yin, G.: Delta wave and vacuum state for generalized Chaplygin gas dynamics system as pressure vanishes. Nonlinear Anal., Real World Appl. 22, 115-128 (2015)

19. Yang, H., Wang, J.: Delta-shocks and vacuum states in the vanishing pressure Imit of solutions to the isentropic Euler equations for modified Chaplygin gas. J. Math. Anal. Appl. 413, 800-820 (2014)

20. Lax, P.D.: Hyperbolic Systems of Conservation Laws and the Mathematical Theory of Shock Waves. SIAM, Philadelphia (1973)

21. Chang, T., Hsiao, L.: The Riemann Problem and Interaction of Waves in Gas Dynamics. Longman, Essex (1989)

22. Smoller, J.: Shock Waves and Reaction-Diffusion Equations, 2nd edn. Springer, New York (1994)

23. Shen, C.: The Riemann problem for the Chaplygin gas equations with a source term. Z. Angew. Math. Mech. 96, 681-695 (2016)

24. Sun, M.: The exact Riemann solutions to the generalized Chaplygin gas equations with friction. Commun. Nonlinear Sci. Numer. Simul. 36, 342-353 (2016)

25. Gu, F., Lu, Y.G., Zhang, Q.: Global solutions to one-dimensional shallow water magnetohydrodynamic equations, J. Math. Anal. Appl. 401, 714-723 (2013)

26. Kraiko, A.N.: Discontinuity surfaces in medium without self-pressure. Prikl. Mat. Mekh. 43, 539-549 (1979)

27. Nilsson, B., Rozanova, O.S., Shelkovich, V.M.: Mass, momentum and energy conservation laws in zero-pressure gas dynamics and delta-shocks: Il. Appl. Anal. 90, 831-842 (2011)

28. Nilsson, B., Shelkovich, V.M.: Mass, momentum and energy conservation laws in zero-pressure gas dynamics and delta-shocks. Appl. Anal. 90, 1677-1689 (2011)

29. Cheng, H.: Riemann problem for one-dimensional system of conservation laws of mass, momentum and energy in zero-pressure gas dynamics. Differ. Equ. Appl. 4, 653-664 (2012)

30. Shelkovich, V.M.: Delta-shock waves in nonlinear chromatography. In: 13th International Conference on Hyperbolic Problems: Theory, Numerics, Applications, June 15-19, Xijiao Hotel, Beijing, China (2010)

31. Shelkovich, V.M.: One class of systems of conservation laws admitting delta-shocks. In: 13th International Conference on Hyperbolic Problems: Theory, Numerics, Applications, June 15-19, Xijiao Hotel, Beijing, China (2010)

32. Cheng, H., Yang, H.: Delta shock waves in chromatography equations. J. Math. Anal. Appl. 380, 475-485 (2011)

33. Yang, H., Zhang, Y.: New developments of delta shock waves and its applications in systems of conservation laws. J. Differ. Equ. 252, 5951-5993 (2012)

34. Yang, H., Zhang, Y.: Delta shock waves with Dirac delta function in both components for systems of conservation laws. J. Differ. Equ. 257, 4369-4402 (2014)

35. Zhu, L., Sheng, W.: The Riemann problem of adiabatic Chaplygin gas dynamic system. Commun. Appl. Math. Comput. 24, 9-16 (2010)

36. Pang, Y.: Delta shock wave in the compressible Euler equations for a Chaplygin gas. J. Math. Anal. Appl. 448, 245-261 (2017)

37. Pang, Y.: Delta shock wave with Dirac delta function in multiple components for the system of generalized Chaplygin gas dynamics. Bound. Value Probl. 2016, Article ID 202 (2016)

38. Faccanoni, G., Mangeney, A.: Exact solution for granular flows. Int. J. Numer. Anal. Methods Geomech. 37, 1408-1433 (2012)

\section{Submit your manuscript to a SpringerOpen ${ }^{\circ}$ journal and benefit from:}

- Convenient online submission

- Rigorous peer review

- Open access: articles freely available online

- High visibility within the field

- Retaining the copyright to your article

Submit your next manuscript at $\boldsymbol{~ s p r i n g e r o p e n . c o m ~}$ 